\title{
Remarks on Framed Bordism Classes of Classical Lie Groups
}

\author{
By
}

Haruo Minami*

\section{Introduction}

We write $(G, R)$ for a compact connected Lie group $G$ of dimension $d>0$ regarded as a framed manifold with the right invariant framing $R$ and denote by $[G, R]$ its bordism class determined in $\pi_{d}^{S}$ via the Thom-Pontrjagin construction. For this it is known $[7]$ that $72[G, R]=0$ and more previously in [2] it is conjectured that $[G, R]=0$ if $\operatorname{rank} G \geq 10$ or so. We have $[S O(2 n), R]=0(n \geq 2)$ already in $[3]$ and so we are interested in such a conjecture for the cases $G=S O(n), S U(n)$ or $S p(n)$.

In this note we consider a slight modification of Proposition 5.3 of [1] which describes the behavior of framings of $G$ and using this we show the following partial results:

$$
\begin{aligned}
& {[S O(8 n+1), R]=0, \quad[S O(32 n+3), R]=0,[S p(8 n), R]=0 \text { and }} \\
& {[S U(8 n+1), R]=0 \quad \text { for } n \geq 1}
\end{aligned}
$$

And also we give a direct proof of the result of [6] about the 3-component of $[S O(2 n+1), R]$ and so of $[S p(n), R]$. In particular, two third parts of it follow immediately from this modification.

\section{$\S 1$. A Formula for Null Bordism Classes}

In this section we will reconsider a result of Proposition 5.3 of [1] and give a proof of it in order to grope for a little improvement. Let $G$ be a compact connected Lie group and $H \subset G$ a closed subgroup isomorphic to $S^{s}$ where

Communicated by K. Saito. Received February 1, 2006.

2000 Mathematics Subject Classification(s): 55N22 (19L20, 57R15).

*Department of Mathematics, Nara University of Education, Takabatake-cho, Nara 630, Japan.

(C) 2007 Research Institute for Mathematical Sciences, Kyoto University. All rights reserved. 
$s=1$ or 3 . Let us assume that $H$ is identified with $S^{s}$ and denote by $\sigma$ the usual inclusion $H \hookrightarrow S O(s+1)$.

Let $\eta$ be the vector bundle associated via $\sigma$ with the principal $H$-bundle $\pi: G \rightarrow G / H$ and denote by $p: D(\eta) \rightarrow G / H$ its disk bundle. Here $G / H$ means the space of right cosets of $H$ in $G$ as usual. Putting $W=D(\eta)$ its tangent bundle $T(W)$ can be decomposed as

$$
T(W) \cong p^{*}(T(G / H) \oplus \eta)
$$

as vector bundles over $W$ where $T(G / H)$ denotes the tangent bundle of $G / H$ (see [2] and also [4]). Clearly the associated sphere bundle $S(\eta) \rightarrow G / H$ of $\eta$ is isomorphic to the principal $H$-bundle $\pi: G \rightarrow G / H$. Therefore we see that the restriction of the equation of (1.1) to $S(\eta)$ becomes an isomorphism

$$
T(G) \oplus(G \times \boldsymbol{R}) \cong \pi^{*}(T(G / H)) \oplus \pi^{*}(\eta)
$$

of vector bundles over $G$ where $T(G)$ denotes the tangent bundle of $G$. Note that $\pi^{*}(\eta)$ has a natural cross-section so that it can be decomposed as $\pi^{*}(\eta) \cong$ $\eta_{0} \oplus(G \times \boldsymbol{R})$. Here $\eta_{0}$ coincides with the bundle $T_{H}(G)$ consisting of tangents along the fibres of the principal $H$-bundle $\pi: G \rightarrow G / H$. This has a natural right action of $H$. So we have an isomorphism

$$
T(G) \oplus(G \times \boldsymbol{R}) \cong \pi^{*}\left(T(G / H) \oplus T_{H}(G) / H\right) \oplus(G \times \boldsymbol{R})
$$

of vector bundles over $G$.

Analogously as in (1.1) we have a decomposition

$$
T(G) \cong \pi^{*}\left(T(G / H) \oplus T_{H}(G) / H\right) .
$$

It is easily checked that the equation of (1.2) is just a stabilization of this isomorphism. Now dividing the equation of (1.3) by the right action of $H$ yields an isomorphism

$$
T(G) / H \cong T(G / H) \oplus T_{H}(G) / H
$$

of vector bundles over $G / H$. Denote by $\operatorname{Ad}_{G}\left(\right.$ resp. $\left.\operatorname{Ad}_{H}\right)$ the adjoint representation of $G$ (resp. $H$ ). Then the restriction of $\operatorname{Ad}_{G}$ to $H$ is decomposed as

$$
\operatorname{Ad}_{G} \mid H=\operatorname{Ad}_{(G, H)} \oplus \operatorname{Ad}_{H}
$$

since $\operatorname{Ad}_{G} \mid H$ contains $\operatorname{Ad}_{H}$ as a subrepresentation. Let $H$ act via $\operatorname{Ad}_{G} \mid H$ on the tangent space $T_{e}(G)$ at the identity element $e$ of $G$. Then $T_{e}(G)$ is 
also decomposed as $T_{e}(G)=V \oplus T_{e}(H)$ corresponding to the decomosition of $\operatorname{Ad}_{G} \mid H$ mentioned above. Consider the isomorphism $L: T(G) \rightarrow G \times T_{e}(G)$ of vector bundles given by $L(v)=\left(g, L_{g^{-1} *}(v)\right)$ for $v \in T_{g}(G)$ where $L_{g^{-1}}$ denotes left multiplication by $g^{-1}$ and $T_{g}(G)$ the tangent space at $g \in G$. Since this isomorphism becomes compatible with the right action of $H$, we have $T(G) / H \cong G \times_{H} T_{e}(G)$. Similarly we obtain $T_{H}(G) / H \cong G \times_{H} T_{e}(H)$ so that we can deduce from the equation following (1.3)

$$
T(G / H) \cong G \times_{H} V .
$$

Let us identify the preceding two isomorphisms and write $g \times{ }_{H} u$ for the element of $G \times{ }_{H} V\left(\right.$ resp. $\left.G \times{ }_{H} T_{e}(H)\right)$ represented by $(g, u) \in G \times V\left(\right.$ resp. $\left.G \times T_{e}(H)\right)$. If furthermore an element $v \in T_{e}(G)$ is decomposed as

$$
v=v_{b}+v_{f}
$$

where $v_{b} \in V$ and $v_{f} \in T_{e}(H)$, then we see that the isomorphism of vector bundles of (1.3) is given by the assignment

$$
L_{g_{*}}(v) \mapsto\left(g \times_{H} v_{b}\right)+\left(g \times_{H} v_{f}\right) .
$$

Here we recall the definition of framings of tangent bundles. Identifying $\boldsymbol{R}^{d}$ with $T_{e}(G)$ in a orientation preserving way, the right invariant framing $R: T(G) \rightarrow G \times \boldsymbol{R}^{d}$ of $T(G)$ is given by $R(v)=\left(g, R_{g^{-1}}(v)\right)$ for $v \in T_{g}(G)$ where $R_{g^{-1}}$ denotes right multiplication by $g^{-1}$. We note here that the left invariant framing $L$ defined above and this one are transformed into each other with the change of orientation by $(-1)^{d}$ in degree under the map $t: G \rightarrow G$ given by $t(g)=g^{-1}$ for $g \in G$, i.e. it holds that $[G, R]=(-1)^{d}[G, L]$.

Given a map $\varphi: G \rightarrow S O(n)$, we have an automorphism of the trivial bundle $G \times \boldsymbol{R}^{n}$ given by $(g, w) \mapsto\left(g, \varphi(g)^{-1}(w)\right)$. Then the twisted framing $R^{\varphi}$ of $R$ by $\varphi$ is defined as a direct sum of $R$ and this automorphism. It is easy to see that the determination of $\left[G, R^{\varphi}\right]$ depends essentially on the element $\beta(\varphi)$ of $\widetilde{K O}^{-1}\left(G^{+}\right)$represented by $\varphi$ where $G^{+}$denotes the $G$ adjoined a disjoint base point. In fact by definition ([10], Proposition 8.14) we see that $\left[G, R^{\varphi}\right]$ can be represented as a Kronecker product of $J(\beta(\varphi)) \in \pi_{S}^{0}\left(G^{+}\right)$and the homotopy fundamental class $\sigma(G, R) \in \pi_{d}^{S}\left(G^{+}\right)$of $(G, R)$, i.e.

$$
\left[G, R^{\varphi}\right]=\langle J(\beta(\varphi)), \sigma(G, R)\rangle
$$

where $J$ denotes the $J$-map $\widetilde{K O}^{-1}\left(G^{+}\right) \rightarrow \pi_{S}^{0}\left(G^{+}\right)$. Henceforth we assume that $\varphi$ is identified with $\beta(\varphi)$. Also we abbreviate $\left(G, R^{\varphi}\right)$ to $(G, \varphi)$ and write $\left[G, R^{\varphi}\right]=[G, \varphi]$ so that we have $[G, 0]=[G, R]$. 
We turn now to the equation of (1.1). Suppose that there is a real representation $f$ of $G$ such that

$$
f \mid H=\operatorname{Ad}_{(G, H)} \oplus \sigma \oplus \ell
$$

where the integer $\ell$ denotes the $\ell$-dimensional trivial representation. Applying $f$ to the equation of (1.1) in the usual fashion under the identification of (1.4) yields an isomorphism

$$
F: T(W) \oplus\left(W \times \boldsymbol{R}^{\ell}\right) \rightarrow W \times \boldsymbol{R}^{d+\ell+1}
$$

of vector bundles over $W$, so that $W$ becomes a framed manifold with $F$ as a framing. And further considering the assignment of (1.5) we find that the restriction

$$
F^{\prime}: T(G) \oplus\left(G \times \boldsymbol{R}^{\ell+1}\right) \rightarrow G \times \boldsymbol{R}^{d+\ell+1}
$$

of $F$ to the restriction of $T(W) \oplus\left(W \times \boldsymbol{R}^{\ell}\right) \rightarrow W$ to $G \subset W$ is given by

$$
(v,(g, w)) \mapsto(g, f(g)(v+w))
$$

for $v \in T_{g}(G)$ and $w \in \boldsymbol{R}^{\ell+1}$. This implies that the twisted framing of $R$ by $-f$ equals $F^{\prime}$, so that it can be extended over $W$. So $(G,-f)$ becomes a framed boundary of $(W, F)$.

Note that this result is slightly generalized as follows. Let $\rho_{1}, \rho_{2}$ be $n$ dimensional real representations of $G$ such that $\rho_{1}\left|H=\rho_{2}\right| H$. Define a map $\bar{\varphi}: G / H \rightarrow S O(n)$ by $\bar{\varphi}(g H)=\rho_{1}(g) \rho_{2}(g)^{-1}$ for $g \in G$ and put $\varphi=\bar{\varphi} \circ p:$ $W \rightarrow S O(n)$. Then we see that the restriction of the twisted framing $F^{\varphi}$ of $W$ to $G$ equals the twisted framing of $R$ by $-f+\rho_{1}-\rho_{2}$, so that $\left(G,-f+\rho_{1}-\rho_{2}\right)$ also becomes a framed boundary of $\left(W, F^{\varphi}\right)$.

In general there holds the following formula. For any real representations $\rho_{1}, \rho_{2}$ of $\mathrm{G}$ we have

$$
\left[G, \operatorname{Ad}_{G}-\rho_{1}+\rho_{2}\right]=(-1)^{d}\left[G, \rho_{1}-\rho_{2}\right] .
$$

This is an easy modification of Lemma 4 of [9]. The proof can be done also using the map $t: G \rightarrow G$ as above. In fact $t$ transforms $\left(G, \operatorname{Ad}_{G}-\rho_{1}+\rho_{2}\right)$ into $\left(G, \rho_{1}-\rho_{2}\right)$ and then changes the orientation by $(-1)^{d}$ in degree. Using (1.7) we therefore have that $\left(G, \operatorname{Ad}_{G}+f-\rho_{1}+\rho_{2}\right)$ is framed null-bordant. Thus we get the following.

Proposition 1.8 (cf. [1], Proposition 5.3). Let $H \subset G$ be a closed subgroup isomorphic to either $S^{1}$ or $S^{3}$. Suppose that there exists a real representation $f$ of $G$ such that $f \mid H=\operatorname{Ad}_{(G, H)} \oplus \sigma$ up to trivial representations 
and given two real representations $\rho_{1}, \rho_{2}$ of $G$ satisfying $\rho_{1}\left|H=\rho_{2}\right| H$. Then

$$
\left[G,-f+\rho_{1}-\rho_{2}\right]=0
$$

(hence equivalently $\left[G, \operatorname{Ad}_{G}+f-\rho_{1}+\rho_{2}\right]=0$ ).

\section{§2. Formulas for Classical Lie Groups}

By (1.6) we have $[G, \varphi]=\langle J(\varphi), \sigma(G, R)\rangle$. Hence putting $\kappa(x)=\langle x$, $\sigma(G, R)\rangle$ for $x \in \pi_{S}^{0}\left(G^{+}\right)$we can write as $\kappa(J(\varphi))=[G, \varphi]$ and in particular $\kappa(1)=[G, R]$ since $J(0)=1$. For any prime $p$ we denote by $x_{(p)}$ the $p$ component of elements $x$ of the relevant groups and further by $x_{(\text {odd })}$ the oddcomponent of $x$. Let $\rho$ denote the identity map representation of $S O(n)$ or the realifications of those of $S U(n)$ and $S p(n)$. Then from Proposition 1.8 we have the following proposition.

Proposition 2.1 (cf. [1], Proposition 5.2).

a) $[S O(n),-(n-1-3 k) \rho]_{(o d d)}=0(n \geq 2)$,

$[S O(n),-(n-3-3 k) \rho]_{(o d d)}=0(n \geq 4)$,

$[S O(n),-(n-1-8 k) \rho]_{(2)}=0(n \geq 2)$,

$[S O(n),-(n-3-32 k) \rho]_{(2)}=0(n \geq 4)$,

b) $[S U(n),-(n-1-3 k) \rho]_{(o d d)}=0(n \geq 2)$,

$[S U(n),-(n-1-8 k) \rho]_{(2)}=0(n \geq 2)$,

c) $[S p(n),-(n-3 k) \rho]_{(o d d)}=0(n \geq 1)$,

$[S p(n),-(n-8 k) \rho]_{(2)}=0(n \geq 1)$

for integer $k$.

Proof. a) Choose $S O(2) \times I_{n-2}$ for the subgroup $H$ of $G=S O(n)$ in Proposition 1.8 where $I_{t}$ denotes the unit matrix of degree $t$. Then we can take $(n-1) \rho$ for the representation $f$ required in Proposition 1.8 because $\operatorname{Ad}_{G}=\lambda^{2} \rho$, $\operatorname{Ad}_{H}=1$ and $\rho \mid H=\sigma \oplus(n-2)$. Moreover, for any $k \geq 0$, if we take $\rho_{1}=k\left(\rho^{2} \oplus\left(n^{2}-3 n\right)\right)$ and $\rho_{2}=k\left(\psi^{2} \rho \oplus(2 n-4) \rho\right)$ where $\psi^{2}$ denotes the 2nd Adams operation, then we see that $\rho_{1}$ coincides with $\rho_{2}$ on $H$. Hence we have from Proposition 1.8

$$
\kappa\left(J\left(-(n-1) \rho-k\left(\psi^{2} \rho-\rho^{2}+(2 n-4) \rho\right)\right)\right)=0 .
$$


for any $k \geq 0$. But interchange $\rho_{1}$ and $\rho_{2}$ shows that this equality is valid in the case where $k<0$. Now the solution of the Adams conjecture [8] shows that $J\left(\rho-\psi^{2} \rho\right)_{(o d d)}=1$ and also $J\left(\rho^{2}-2 n \rho\right)=1$ since $\beta\left(\rho^{2}\right)=2 n \beta(\rho)$ in $\widetilde{K O}^{-1}\left(G^{+}\right)$. Hence by substituting these two equalities into the above one using the multiplicative formula $J(x+y)=J(x) J(y)$, we get for any integer $k$

$$
\kappa(J(-(n-1-3 k) \rho))_{(o d d)}=0
$$

which shows that $[S O(n),-(n-1-3 k) \rho]_{(o d d)}=0$.

To prove the second formula we set $H=S U(2) \times I_{n-4} \subset G=S O(n)$. Then we can take $f=(n-3) \rho$ and also it can be verified that $2 \psi^{2} \rho \oplus(2 n-8) \rho$ coincides with $\rho^{2} \oplus\left(n^{2}-6 n\right)$ on $H$. So by applying Proposition 1.8 again we have

$$
\kappa\left(J\left(-(n-3) \rho-k\left(\left(1+p^{N}\right) / 2\right)\left(2 \psi^{2} \rho-\rho^{2}+(2 n-8) \rho\right)\right)\right)=0
$$

for any integer $k$ and $N \geq 0$ where $p$ denotes an odd prime. Hence by the same reason as above we have

$$
\kappa\left(J\left(-(n-3-3 k) \rho+3 k p^{N} \rho\right)\right)_{(o d d)}=0 .
$$

Now we know that $J(x) \in \pi_{S}^{0}\left(G^{+}\right)=\boldsymbol{Z} \oplus \pi_{S}^{0}(G)$ can be written as $J(x)=$ $1+\tilde{J}(x)$ where $\tilde{J}(x) \in \pi_{S}^{0}(G)$ and any elment of $\pi_{S}^{0}(G)$ is nilpotent and has finite order. So if $N$ is taken to be sufficiently large, then we see that there holds $J\left(3 k p^{N} \rho\right)_{(p)}=1$. Therefore it follows from the above equality that $\kappa\left(J(-(n-3-3 k) \rho)_{(p)}=0\right.$ for any odd prime $p$, so that we have

$$
\kappa(J(-(n-3-3 k) \rho))_{(o d d)}=0
$$

and hence $[S O(n),-(n-3-3 k) \rho]_{(o d d)}=0$ for any integer $k$.

As for the succeeding two formulas about their 2-components it suffices to use the 3rd Adams operation $\psi^{3}$ instead of $\psi^{2}$ in the arguments similar to those in the above cases.

b), c) The proofs of these two cases are quite parallel to that of a). Here it suffices to choose $S U(2) \times I_{n-2}$ (resp. $\left.S p(1) \times I_{n-1}\right)$ for the required subgroup $H$ of $G=S U(n)$ (resp. $S p(n))$. Then $f$ can be taken to be $(n-1) \rho($ resp. $n \rho)$.

\section{Proposition 2.2.}

a) $[S O(3 n+1), R]_{(o d d)}=0$, 


$$
\begin{aligned}
& {[S O(3 n+3), R]_{(\text {odd })}=0, } \\
& {[S O(8 n+1), R]_{(2)}=0, } \\
& {[S O(32 n+3), R]_{(2)}=0, } \\
& \text { b) } \quad[S U(3 n+1), R]_{(o d d)}=0, \\
& \text { c) } \quad[S P(3 n), R]_{(\text {odd })}=0, \\
& {[S p(8 n), R]_{(2)}=0 }
\end{aligned}
$$

for $n \geq 1$.

Proof. These follow by substituting adequate integers for $n$ and $k$ in the formulas of Proposition 2.1. For example, the first formula is just the first one of Proposition 2.1 with $3 n+1$ instead of $n$ and $k=n$.

The following is also an immediate corollary of Proposition 2.2.

\section{Corollary 2.3.}

$$
\begin{aligned}
& {[S O(24 n+1), R]=0,} \\
& {[S O(96 n+3), R]=0,} \\
& {[S U(24 n+1), R]=0,} \\
& {[S p(24 n), R]=0}
\end{aligned}
$$

for $n \geq 1$.

As noted in the introduction we know in $[7]$ that $[G, R]$ has at most 2and 3-components. But we have $[G, R]_{(3)}=0$ for $G=S O(2 n+1), S p(n)(n \geq$ $3, n \neq 5,7,11)$ and $S U(n)(n \geq 3)$ by [6] and [5] respectively. Hence Corollary 2.3 can be improved as follows.

\section{Corollary 2.4.}

$$
\begin{aligned}
& {[S O(8 n+1), R]=0,} \\
& {[S O(32 n+3), R]=0,} \\
& {[S U(8 n+1), R]=0,}
\end{aligned}
$$




$$
[S p(8 n), R]=0
$$

for $n \geq 1$.

\section{§3. Remarks for $S O(2 n+1)$ and $S p(n)$}

In this section we will give a direct proof of the following result of [6] using Proposition 2.1.

$$
[S O(2 n+1), R]_{(3)}=0, \text { equivalently }[S p(n), R]_{(3)}=0 \text { for } n \geq 3, n \neq 5
$$

Here we find that this assertion holds in the cases $n=7$ and 11 which remain undecided in [6]. But it is regrettable that the case $n=5$ does so yet. Since $[S O(2 n+1), R]_{(3)}$ and $[S p(n), R]_{(3)}$ have the same order by Lemma 2.6 of $[6]$ we consider only the case $G=S O(2 n+1)$ below.

Especially the cases where $n \equiv 0,1 \bmod 3$ are straightforward from the first and second formulas of Proposition 2.1, a). In fact by substituting $n=$ $3 \ell, k=2 \ell$ and $n=3 \ell+1, k=2 \ell$ into them with $2 n+1$ instead of $n$ respectively we can get $[G, R]_{(3)}=0$ immediately.

We next consider the case $n \equiv 2 \bmod 3$, i.e. $n=3 \ell+2(\ell \geq 2)$. Putting $\mu=\tilde{J}(\rho)_{(3)}$ where $\rho$ is as above we have from the first and second formulas of Proposition 2.1, a)

$$
\kappa\left((1+\mu)^{3 k+1}\right)=0 \quad \text { and } \quad \kappa\left((1+\mu)^{3 k+2}\right)=0 \quad \text { for } \quad k \geq 0 .
$$

Let $H=S O(2) \times I_{2 n-1} \subset G$ and let $G$ and $T(G)$ be provided with the natural right actions of $H$. Then the twisted framing $R^{\varphi}$ which occurs in (1.6) becomes an $H$-equivariant isomorphism. So we can apply Lemma 2.2 of [7] to this equivariant framed manifold $\left(G, R^{\varphi}\right)$ and hence we have $24[G, \varphi]=0$ by the same argument as in Theorem 1.1 of [7]. So taking as $\varphi$ the one used in the proof of Proposition 2.1, a) we have $3 \kappa\left((1+\mu)^{3 k}\right)=0$ for any $k \geq 0$. Combining this with (3.2) yields $3 \kappa\left((1+\mu)^{k}\right)=0$ for any $k \geq 0$ and so by induction on $k$ we have

$$
3 \kappa\left(\mu^{k}\right)=0 \quad \text { for } k \geq 0 .
$$

Using this Theorem (5.3) of [2] gives

$$
[G, R]_{(3)}=(-1)^{\ell} \kappa\left(\mu^{3 \ell+2}\right) \quad \text { and } \quad \mu^{3 \ell+3}=0 .
$$

From now on we work modulo 3 due to (3.3). Again from (3.2) by induction on $k$ we have

$$
\kappa\left(\mu^{3 k}+\mu^{3 k+1}\right)=0 \quad \text { and } \quad \kappa\left(\mu^{3 k+1}+\mu^{3 k+2}\right)=0 \quad \text { for } \quad k \geq 0 .
$$


Put $\alpha=\tilde{J}\left(\operatorname{Ad}_{G}\right)_{(3)}$. Then it can be deduced from (1.7) that

$$
\kappa\left((1+\alpha) \mu^{k}\right)=(-1)^{\ell} \kappa\left(R^{k}\right) \text { and } \kappa\left((1+\alpha) R^{k}\right)=(-1)^{\ell} \kappa\left(\mu^{k}\right) \text { for } k \geq 0
$$

where $R$ is the formal power series given by $1+R=(1+\mu)^{-1}$. As is seen above it holds that $J\left(\rho-\psi^{2} \rho\right)_{(3)}=1$ so that $J\left(2 \lambda^{2} \rho\right)_{(3)}=J((12 \ell+9) \rho)_{(3)}$. Since $\lambda^{2} \rho=\operatorname{Ad}_{G}$ this yields

$$
1+\alpha=1-\ell \mu^{3}-\ell(\ell+1) \mu^{6}+(\ell+1)\left(\ell^{2}-\ell+3\right) / 3 \mu^{9}-\ell^{2}(\ell+1)\left(\ell^{2}-\ell+3\right) / 3 \mu^{12}+h_{15}
$$

where $h_{15}$ denotes the sum of the higher terms with degrees above 15 . From (3.5) it follows immediately that

$$
\begin{gathered}
\kappa\left(\mu^{3 \ell+2}\right)=-\kappa\left(\mu^{3 \ell+1}\right)=\kappa\left(\mu^{3 \ell}\right), \kappa\left(\mu^{3 \ell-1}\right)=-\kappa\left(\mu^{3 \ell-2}\right)=\kappa\left(\mu^{3 \ell-3}\right), \\
\kappa\left(\mu^{3 \ell-4}\right)=-\kappa\left(\mu^{3 \ell-5}\right)=\kappa\left(\mu^{3 \ell-6}\right), \kappa\left(\mu^{3 \ell-7}\right)=-\kappa\left(\mu^{3 \ell-8}\right)=\kappa\left(\mu^{3 \ell-9}\right) .
\end{gathered}
$$

The calculations below are done taking account of these equalities. By calculating both of the equalities of (3.6) with $k=3 \ell-6$ we obtain

$$
\kappa\left(-\mu^{3 \ell-3}+(\ell+1) \mu^{3 \ell}\right)=0 \quad \text { and } \quad \kappa\left((\ell-1) \mu^{3 \ell-3}+\left(\ell^{2}+1\right) \mu^{3 \ell}\right)=0 .
$$

These show that $\kappa\left(\mu^{3 \ell+2}\right)=0$. Hence by virtue of (3.4) we see that if $\ell$ is prime to 3 then $[G, R]_{(3)}=0$. Now suppose that $\ell \equiv 0 \bmod 3$ and put $\ell=3 \mathrm{~s}$. Then calculating the first equalities of (3.6) with $k=3 \ell-7,3 \ell-8$ yields

$$
\kappa\left(\mu^{3 \ell-7}-(s-1) \mu^{3 \ell+2}\right)=0 \quad \text { and } \quad \kappa\left(-\mu^{3 \ell-7}+s \mu^{3 \ell+2}\right)=0 .
$$

By adding these equations, we have $\kappa\left(\mu^{3 \ell+2}\right)=0$ which completes the proof of (3.1).

\section{References}

[1] L. Astey, M. A. Guest and G. Pastor, Lie groups as framed boundaries, Osaka J. Math. 25 (1988), no. 4, 891-907.

[2] J. C. Becker and R. E. Schultz, Fixed-point indices and left invariant framings, in $G e-$ ometric applications of homotopy theory (Proc. Conf., Evanston, Ill., 1977), I, 1-31, Lecture Notes in Math., 657, Springer, Berlin.

[3] M. Kamata and H. Minami, The special orthogonal groups $S O(2 n)$ as framed boundaries, Kyushu J. Math. 54 (2000), no. 1, 147-153.

[4] P. Löffler and L. Smith, Line bundles over framed manifolds, Math. Z. 138 (1974), 35-52.

[5] H. Minami, On the 3-component of $S U(n)$ as a framed manifold, Kyushu J. Math. 55 (2001), no. 1, 183-187. 
[6] H. Minami, On framed cobordism classes of classical Lie groups, J. Math. Soc. Japan 55 (2003), no. 4, 1033-1052.

[7] E. Ossa, Lie groups as framed manifolds, Topology 21 (1982), no. 3, 315-323.

[8] D. Quillen, The Adams conjecture, Topology 10 (1971), 67-80.

[9] B. Steer, Orbits and the homotopy class of a compactification of a classical map, Topology 15 (1976), no. 4, 383-393.

[10] R. M. W. Wood, Framing the exceptional Lie group $G_{2}$, Topology 15 (1976), no. 4, 303-320. 